\title{
Revista Brasileirä Multidisciplinar

\section{Estudo da ASSOCIAÇÃo ENTRE PAdrão MASTigatório, Simetria FaCial, disfunÇão TEMPOROMANDIBULAR E POSTURA CORPORAL}

Renata Michele Castilho*; Gabriela de Oliveira Teixeira Castilho*; Karina Dela Coleta Pizzol*; Giovanna Monteiro do Pinho Orlando ${ }^{* *}$; Andrea Carrascosa ${ }^{* * *}$; Ana Lúcia Franco-Michelonii ${ }^{* * *}$; Nadia Lunardi ${ }^{* * *}$.

* Mestre em Ciências Odontológicas Universidade de Araraquara.

${ }^{*}$ Cirurgiã Dentista Universidade de Araraquara.

*** Doutora em Ciências e Tecnologia de Alimentos Universidade de Araraquara.

**** Mestre e Doutora em Reabilitação Oral. Universidade de Araraquara.

${ }_{* * * * *}$ Mestre em Ortodontia e Doutora em Materiais Dentários. Especialista em Ortodontia Universidade de Araraquara.

*Autor para correspondência e-mail:nkpizzol@ig.com.br

\section{Palavras-chave}

Mastigação

Síndrome da disfunção da articulação temporomandibular Postura

Assimetria facial

\section{KEYWORDS}

Chewing

Temporomandibular Joint

Disfunction Syndrome

Posture

Facial Asymmetry

\section{RESUMO}

O estudo objetivou estudar a associação do padrão mastigatório com simetria facial, disfunção temporomandibular (DTM) e postura corporal. Foram selecionados 40 alunos de Odontologia, de ambos os gêneros (idade 18-30 anos). Os critérios de inclusão foram: oclusão normal, bom estado de saúde geral, ausência de patologias crônicas, ausência de discrepâncias esqueléticas, boa saúde periodontal e dentária. Os participantes foram submetidos à avaliação do padrão mastigatório, da postura de ombros e cabeça, análise da simetria facial, análise funcional da oclusão e presença de DTM. A identificação do padrão mastigatório foi realizada por três examinadores treinados, utilizando o método da observação direta associada a imagens de vídeo. Foram registrados: lateralidade (guia canino ou função em grupo) e ângulo funcional mastigatório de Planas (AFMP). Para análise da DTM foi utilizado o RCD/TMD. As avaliações de postura de ombros e da simetria facial foram realizadas por meio de fotografias. Os resultados mostraram que 15 indivíduos apresentaram mastigação unilateral e 25 bilateral. Foi encontrada forte correlação entre o padrão mastigatório e a postura de ombro (coeficiente de contingência $\mathrm{CC}=0,510)$, de cabeça $(\mathrm{CC}=0,673)$ e também com o AFMP $(\mathrm{CC}=0,747)$. Os diagnósticos de desarranjos de disco $(\mathrm{p}=0,0378)$ e desvio da linha média $(\mathrm{p}=0,0111)$ apresentaram diferença estatística nas frequências relatadas entre os grupos, sendo o padrão "normal" mais frequente nos pacientes com mastigação bilateral. Conclui--se que a mastigação unilateral apresenta uma associação com a presença de sinais e sintomas de DTM, com desvios na linha média e com alterações na postura de ombro, cabeça e AFMP.

\section{STUDY OF THE ASSOCIATICON BETWEEN MASTICATORY PATTERM, TEMPOROMADIBULAR DISORDER AND BODY POSTURE}

The study aimed to study the association of masticatory pattern with facial symmetry, temporomandibular disorder (TMD) and body posture. 40 dentistry students of both genders (age 18-30 years) were selected. Inclusion criteria were: normal occlusion, good general health, absence of chronic pathologies, absence of skeletal discrepancies, good periodontal and dental health. The participants underwent an evaluation of the chewing pattern, posture of shoulders and head, analysis of facial symmetry, functional analysis of occlusion and the presence of TMD. The masticatory pattern was identified by three trained examiners, using the direct observation method associated with video images. Laterality (canine guide or group function) and masticatory functional Planas' angle (MFPA) were recorded. For DTM analysis, the RDC/TMD was used. Shoulder posture and facial symmetry evaluations were performed using photographs. The results showed that 15 individuals had unilateral chewing and 25 bilateral. A strong correlation was found between the masticatory pattern and the shoulder posture (contingency coefficient $C C=0.510$ ), head $(C C=0.673)$ and also with the MFPA $(\mathrm{CC}=0.747)$. The diagnoses of disc derangements $(\mathrm{p}=0.0378)$ and deviation from the midline $(\mathrm{p}$ $=0.0111$ ) showed a statistical difference in the reported frequencies between the groups, with the "normal" pattern being more frequent in patients with bilateral chewing. It is concluded that unilateral chewing presents an association with the presence of TMD signs and symptoms, with deviations in the midline and with changes in shoulder, head and MFPA posture. 


\section{INTRODUÇÃo}

A mastigação, é realizada por movimentos mandibulares sincrônicos, controlados pelo complexo sensório-motor trigeminal localizado no tronco encefálico, associado ao feedback de mecanoceptores aferentes presentes nos ligamentos periodontais e fusos musculares, os quais fornecem orientação aos músculos durante a mastigação (VAN et al., 2006). O movimento mastigatório ideal deve ser bilateral equilibrado, com distribuição homegênea entre os ciclos, não apresentando diferenças nas atividades musculares entre os lados (RILO et al., 2009). A trajetória descrita pelo movimento mandibular durante a mastigação tem um formato semelhante à uma gota, sendo esta descrita no sentido horário quando o golpe mastigatório é realizado do lado direito e anti-horário quando do lado esquerdo (SOBOLEVA et al., 2005). O padrão mastigatório pode ser alterado quanto ao perímetro e velocidade dos ciclos de acordo com o tamanho e resistência do bolo alimentar (MIYAWAKI et al., 2001; BHATKA et al., 2004; RILO et al., 2009). O ciclo mastigatório é influenciado pela idade, tipo facial (UEDA., 2006; RILO et al., 2009) e alguns aspectos oclusais como a guia anterior (FERRARIO et al., 2001) e a curvatura de oclusão (YAMAGUCHI et al., 2011).

O sistema estomatognático é um componente importante da parte superior do corpo, composto basicamente pela cabeça, pescoço e cintura escapular, onde, ATM, músculos, ligamentos, fáscias, bem como conexão neural e inervações circulatórias estão todos intimamente relacionados (KHAN et al., 2003). A postura ereta da cabeça é mantida por uma tensão equilibrada entre ossos craniocervicais, estruturas miofasciais e oclusão dentária, sendo que durante a mastigação músculos da cabeça e pescoço demonstram atividade eletromiográfica, o que corrobora o envolvimento dessas estruturas no processo (RILO et al., 2009). A região cervical da coluna é o mediador entre a cabeça e o tronco formando um sistema funcionalmente interligado. A interligação neuroanatômica entre a região bucal e cervical (MICHELOTTI et al., 2011) e a existência das cadeias músculo faciais contribuem na interrelação da cabeça com o restante do corpo humano.

Diante de tal complexidade e interligações, pressupõem-se que alterações funcionais ou estruturais no sistema mastigatório possam, direta ou indiretamente, repercutir em alterações corporais (HIGILEH, 2019), ou seja, desajustes em um dos sistemas leva a reorganização e/ou desajustes em outro sistema visando o menor gasto energético corporal (BEZERRA, 2018). A mastigação unilateral exclusiva ou com predominância unilateral proporciona uma atividade muscular assimétrica, que pode desencadear alterações tanto estruturais como funcionais (RILO et al., 2009; FEDIV, 2017), podendo ser de maior ou menor importância dependendo da frequência, da intensidade, da duração da disfunção e da fase de crescimento do indivíduo.

A mastigação unilateral é relatada na literatura como um fator de alteração da morfologia craniocervical, assim como um fator predisponente das DTM (MIYAKE et al., 2004), uma vez que há uma sobrecarga e concomitante ação de microtraumas na região posterior da ATM contralateral ao lado de trabalho. Além disso, há uma hiperatividade muscular ipsilateral dos músculos da mastigação e também dos músculos posturais, que estão sincronizados (CAMPOS et al., 2008).

Autores como KOHNO, YOSHIDA e KOBAYASHI (1988) e KIBANA, ISHIJIMA e HIRAI (2002) afirmaram em seus artigos que o esternocleidomastóideo, um dos principais músculos responsáveis pela postura da cabeça, está em sincronia com os músculos mastigatórios. Assim, a hiperfunção dos músculos da mastigação, quando mantida por longo período de tempo, poderia ocasionar também uma hipertrofia dos músculos posturais, trazendo como consequência, uma inclinação da cabeça para o mesmo lado.

Nos últimos anos, diversos estudos avaliaram a relação existente entre mastigação unilateral, DTM e alterações dentofaciais (KUMAI et al., 2009; FELICIO et al., 2010; SANTANA-MORA et al., 2013; LAMONTAGNE, AL-TARAKEMAH e HONKALA, 2013; RAVIRA-LASTRA et al., 2014; RAVIRA-LASTRA et al., 2016; PASINATO et al., 2016; PASINATO et al., 2017; TIWARI, NAMBIAR e UNNIKRISHNAN, 2017; KURNIA et al., 2018; LA TOUCHE et al., 2019; FASSICOLLO, 2020). A redução da capacidade 
auditiva unilateral (SANTANA-MORA et al., 2013), inclinação do plano oclusal e desgastes assimétricos das guias (FERRARIO et al., 2001; PIGNATARO et al., 2004) também foram descritos como apresentando correlação positiva com o padrão mastigatório. Em estudos envolvendo animais (POIKELA et al., 1997; POIKELA et al., 2000), induziram a mastigação unilateral em coelhos em fase de crescimento e verificaram alteração mandibular, envolvendo o formato da fossa glenóide, além de alterações histoquímicas que comprometeram a simetria facial.

D’ATTILIO et al. (2005) delinearam uma pesquisa para verificar a possibilidade de induzir experimentalmente alterações no alinhamento da coluna vertebral, em ratos, como conseqüência de alteração na oclusão dental. Os resultados mostraram que todos os ratos do grupo experimental desenvolveram curvas escorióticas e nenhuma alteração naqueles do grupo-controle. Os autores concluíram que o alinhamento da coluna vertebral parece influenciado pela oclusão dental. Essas funções irregulares poderiam criar, a longo prazo, uma série de sinais e sintomas de DTM, com alterações na assimetria facial e na morfologia óssea.

Diante desse contexto, este estudo teve como finalidade avaliar a associação entre mastigação unilateral, assimetria facial, DTM e postura corporal.

\section{Material e Métodos}

Esse estudo foi aprovado pelo Comitê de Ética em pesquisa com Seres Humanos da Universidade de Araraquara (CEP), sob número CAAE 44487015.2.0000.5383, parecer 1.373.594.

\section{Amostra}

Foram selecionados 40 alunos com idade entre 18 e 30 anos, de ambos os gêneros, provenientes dos cursos de graduação e pós-graduação em de Odontologia da Universidade de Araraquara - UNIARA.

Os critérios de inclusão foram: oclusão normal (Classe I de Angle com overjet e overbite entre 2 e $5 \mathrm{~mm}$ ), boa saúde periodontal e dentária e bom estado de saúde geral. Os critérios de exclusão foram: traumas na região crânio-cervical, deformidades congênitas ou neurológicas, discrepância esqueléticas dento-faciais, patologias crônicas como fibromialgia, estar sob tratamento fisioterápico.

Os indivíduos selecionados foram submetidos à avaliação do padrão mastigatório, postura de ombros, análise da simetria facial, análise funcional da oclusão e verificação da presença de DTM, detalhadamente descritos a seguir.

\section{IDENTIFICAÇÃo dO PADRÃo MASTIGATÓRIO}

A identificação do padrão mastigatório foi realizada por três examinadores devidamente treinados, utilizando o método da observação direta associada à imagens de vídeos. Durante a avaliação, o indivíduo permaneceu sentado confortavelmente, mantendo o plano de Frankfurt paralelo ao solo durante a avaliação mastigatória. Uma filmadora digital Canon EOS Rebel T3 (Tóquio, Japão) foi posicionada com o auxilio de um tripé ajustado a cada individuo de acordo com sua estatura, mantendo uma distância padrão de um metro e meio entre os mesmos. Durante a gravação, os indivíduos foram orientados a mastigar 3 balas de goma (3g) e 3 amêndoas (2g) até a deglutição; e 1 goma de mascar Trident (2g) por aproximadamente 20 segundos. Entre um alimento e outro foi permitida ingestão de água e um descanso de 1 minuto. Durante o procedimento, os examinadores avaliaram e classificaram o padrão mastigatório em: mastigação bilateral, mastigação unilateral direita ou mastigação unilateral esquerda.

Análise funcional da oclusão

Por meio de análise clínica e fotográfica, os indivíduos foram avaliados quanto ao tipo de lateralidade (guia canino ou função em grupo) e mensuração do Ângulo Funcional Mastigatório de Planas (AFMP), que consiste no ângulo formado pelo aumento da dimensão vertical (direita e esquerda) e o plano horizontal durante a lateralidade. Esta angulação foi mensurada nas fotos oclusais com a utilização do programa 
Image J (National Institutes of Health, Bethesda, USA) por um único examinador treinado (Figura 1).

Figura 1- Mensuração do AFMP, demonstrando o lado direito com menor ângulo.
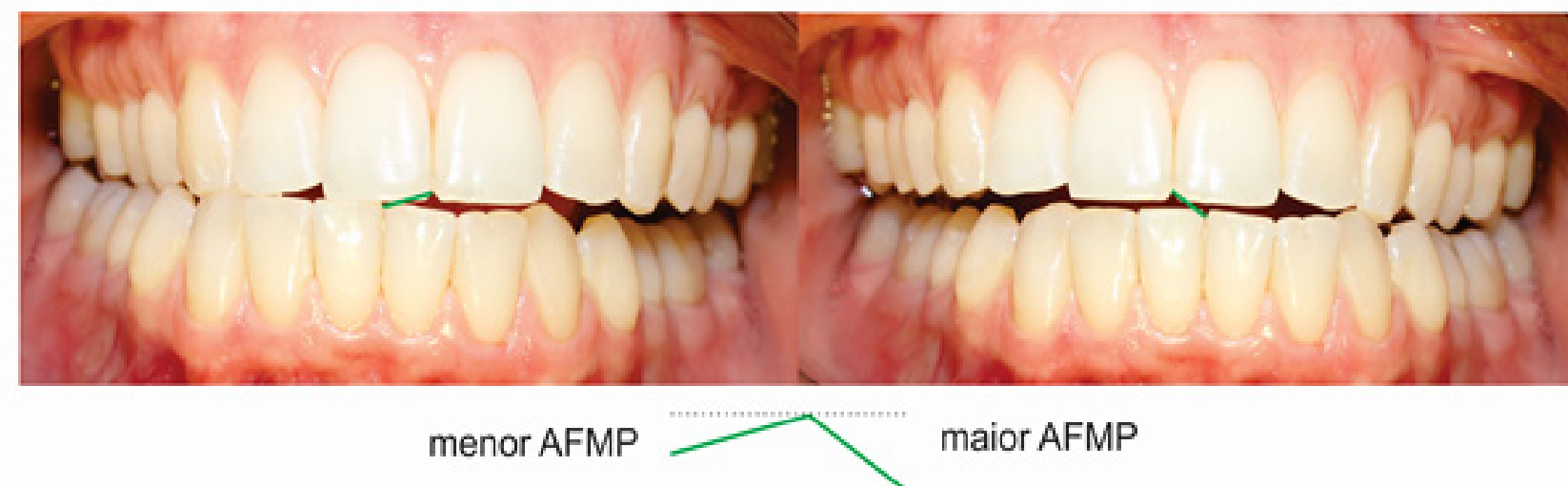

Fonte: Autoria Própria.

\section{ANÁlise da Simetria FACIAL}

Para a análise da simetria facial, o indivíduo foi posicionado, seguindo o padrão previamente descrito para avaliação mastigatória e orientado a permanecer sentado confortavelmente em posição natural de cabeça e olhando para o horizonte. Foram tomadas fotografias faciais frontal com o auxílio de uma câmera fotográfica digital Canon EOS Rebel T3 (Tóquio, Japão) ajustada de acordo com o nível da face de cada individuo.

As imagens foram trabalhadas no programa Image J (National Institutes of Health, Bethesda, USA), utilizando as seguintes linhas de referência: linha mais superior que passa pelo lábio inferior (comissura labial) e plano pupilar. Foram empregadas as grandezas lineares: altura pupilar direita e esquerda pela mensuração da distância de cada pupila ao plano labial inferior (Figura 2). Os pacientes que apresentaram alterações nas proporções mensuradas foram classificados como assimétricos.

Figura 2 - Mensuração da altura pupilar direita e esquerda de um paciente que apresentava leve aumento da altura pupilar esquerda (em vermelho) em relação à direita.

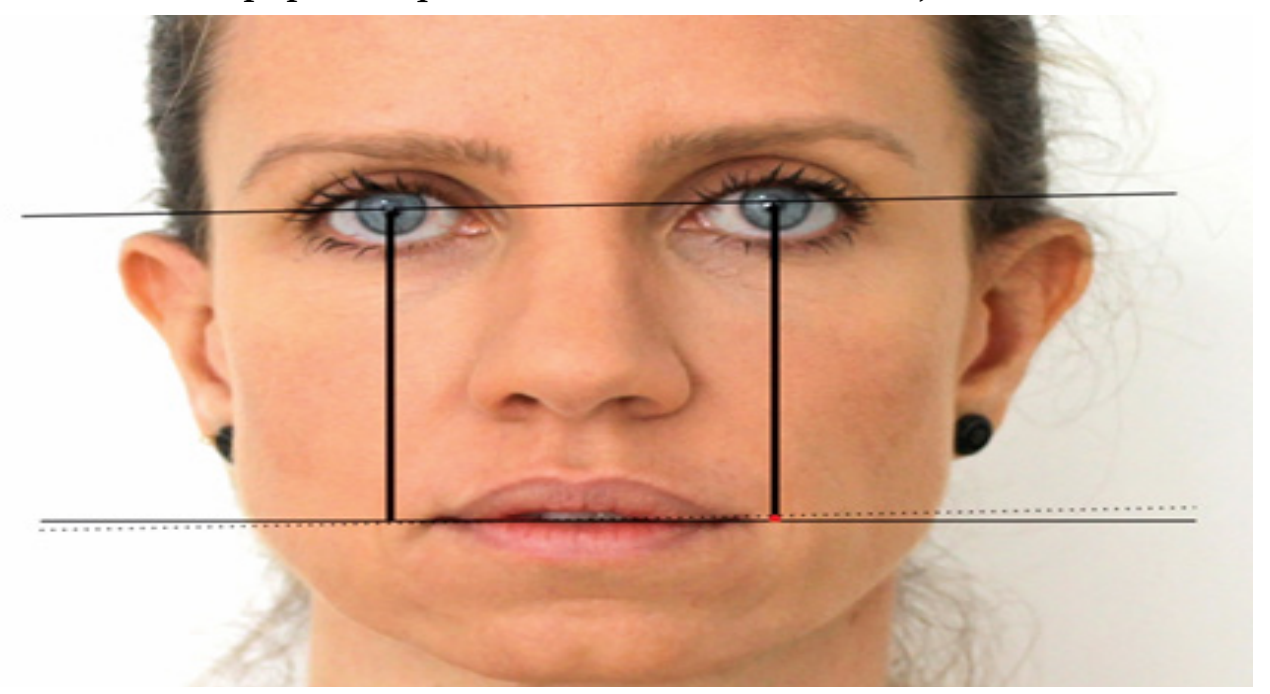

Fonte: Autoria Própria. 


\section{INCLINAÇÃo DOS OMBROS}

Para a avaliação da inclinação dos ombros, em norma frontal, foram tomadas imagens fotográficas dos pacientes de corpo inteiro em posição ortostática natural, mantendo a linha pupilar paralela ao solo. A câmera fotográfica digital Canon EOS Rebel T3 (Tóquio, Japão) foi mantida a uma distância de três metros em relação aos voluntários e altura proporcional ao umbigo do mesmo. As fotografias foram realizadas em ambiente iluminado, tomando como guia um peso vertical para alinhamento da imagem.

As imagens foram recortadas na área de cabeça e ombro e ampliadas para tornar a análise mais precisa. As mensurações foram também realizadas no programa Image J (National Institutes of Health, Bethesda, USA), utilizando as seguintes linhas de referência: linha paralelo ao solo e linha do ombro. A inclinação do ombro foi mensurada em graus pela angulação criada entre a altura dos ombros direito e esquerdo e o plano horizontal (Figura 3).

Figura 3 - Mensuração da diferença de altura de ombro, demonstrando paciente com ombro direito mais alto que o esquerdo.

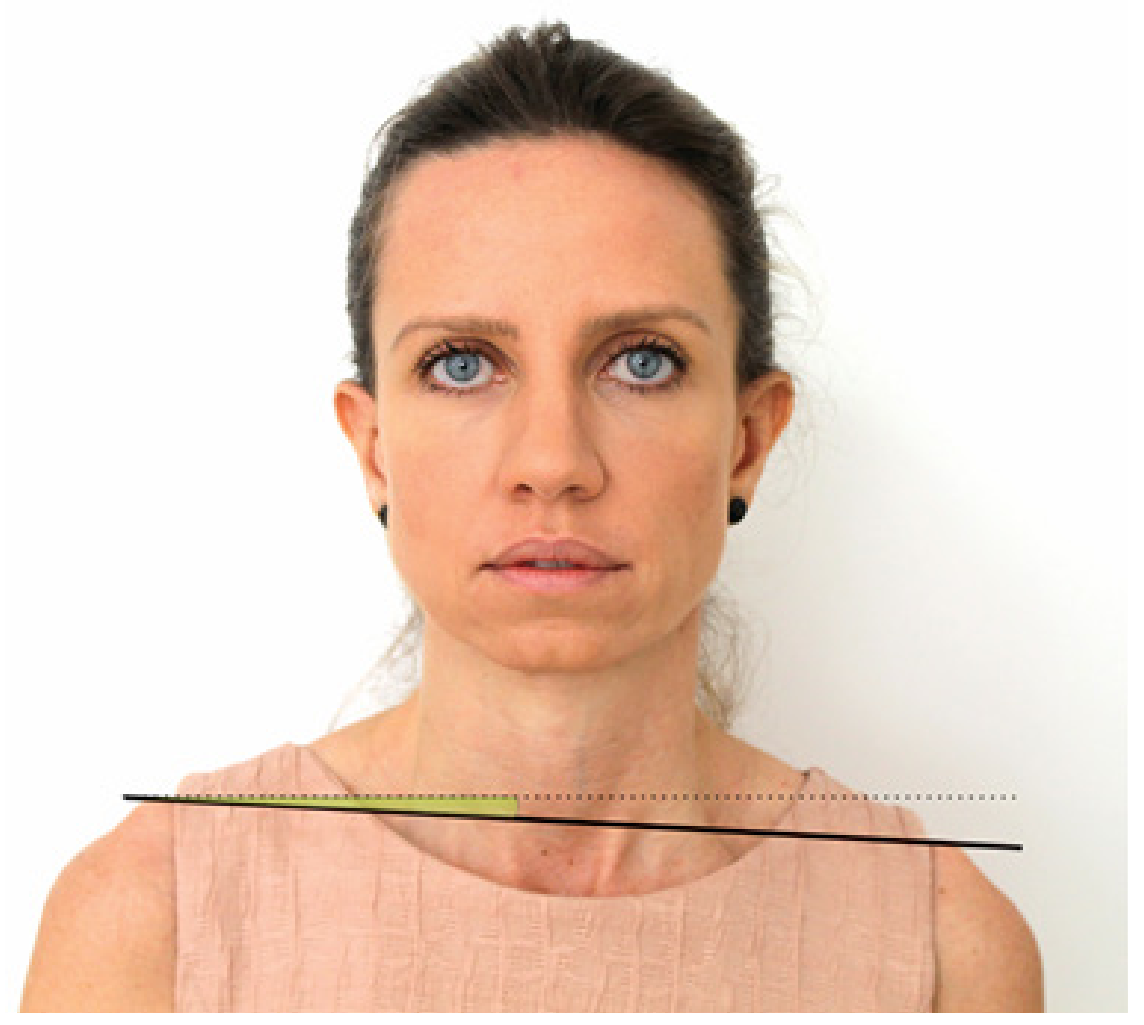

Fonte: Autoria Própria.

\section{INCLINAÇÃO DA CABEÇA}

A mesma imagem fotográfica foi utilizada para verificação do lado de inclinação da cabeça. Cada paciente foi avaliado quanto ao lado da inclinação da cabeça. Esta análise foi realizada utilizando como linhas de referência o plano horizontal, uma perpendicular ao plano horizontal e a linha média da face e verificada qual lado a linha média da face está desviada em relação à perpendicular ao plano horizontal. Os pacientes foram classificados em bem posicionado, inclinado para direita e inclinada para esquerda. 
Figura 4 - Verificação do lado da inclinação da cabeça.

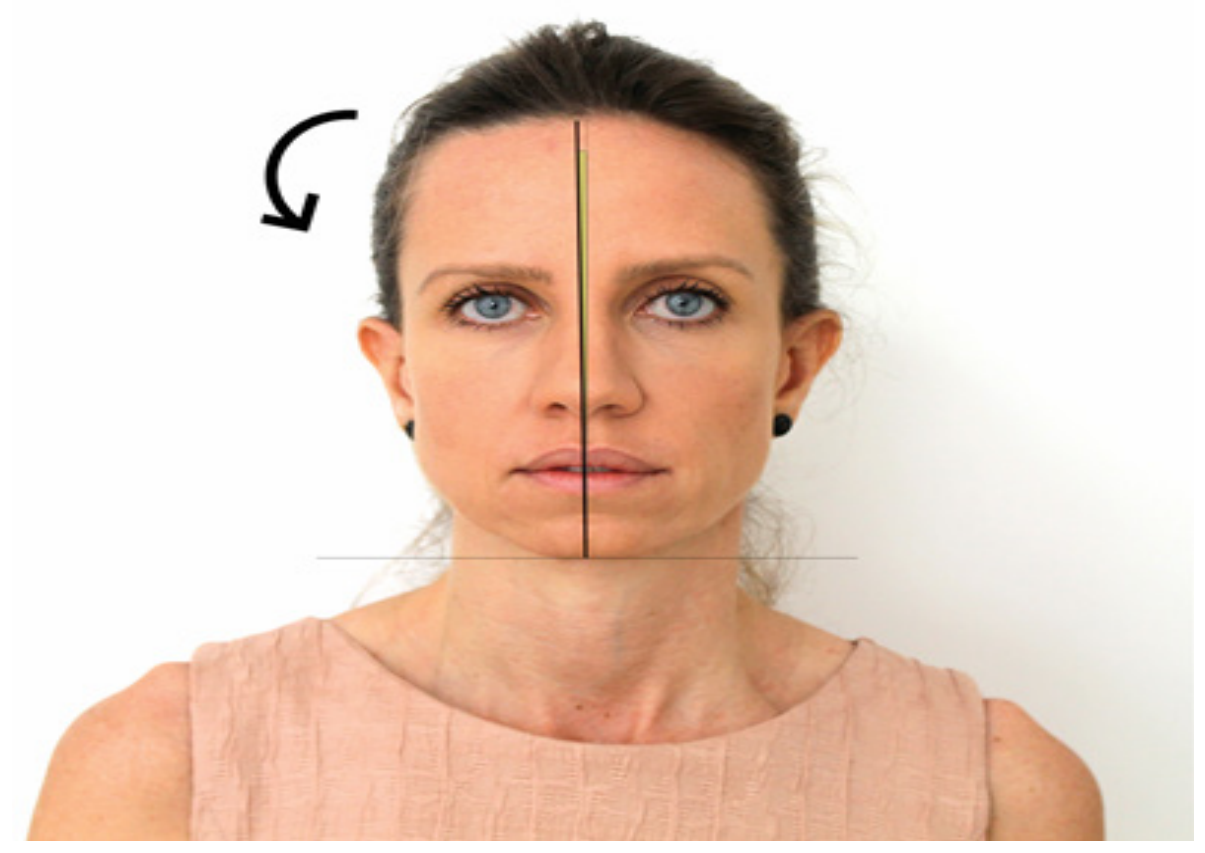

Fonte: Autoria Própria.

\section{Verificação da PResença de Disfunção Temporomandibular (DTM)}

Para o diagnostico de DTM, foram utilizados os Critérios de Diagnostico para pesquisa das Disfunções Temporomandibulares (Research Diagnostic Criteria for Temporomandibular Disorders-RDC/TMD (DWORKIN e LERESCHE, 1992), em sua versão em português (PEREIRA et al., 2004). Para a avaliação da gravidade da dor relacionada à DTM, foi usada a classificação do Eixo II que caracteriza a graduação da dor da DTM (item II.1), os sintomas psicológicos e a presença de depressão (item II.2), outros sintomas físicos não específicos (item II.3), além de limitações relacionadas à função mandibular (item II.4)

\section{ANÁlise dos dAdos}

Para a caracterização da amostra foi utilizada estatística descritiva. As variáveis qualitativas foram apresentadas em frequências simples e relativas (percentuais).

Os valores obtidos foram tabelados no programa Microsoft ${ }^{\circ}$ Excel (versão 16.37) e as análises estatísticas, realizadas no programa SPSS Statistics. Para a verificação de associações entre as variáveis foram realizados o teste do qui-quadrado (adotando o nível de significância de 5\%), coeficiente de contingência e o teste $\mathrm{t}$ (adotando o nível de significância de 5\%).

\section{RESUlTADOS}

De um total de 40 pacientes avaliados: 15 apresentavam mastigação unilateral (38\%, com predomínio do lado direito) e 25 mastigação bilateral (62\%); 40\% era do gênero masculino e 60\% do gênero feminino.

A distribuição dos valores das mensurações pupilares e da inclinação do ombro entre os grupos mastigação unilateral e bilateral está descrita nas figuras 5 e 6 . 
Figura 5 - Análise da mensuração linear da distância da altura pupilar.

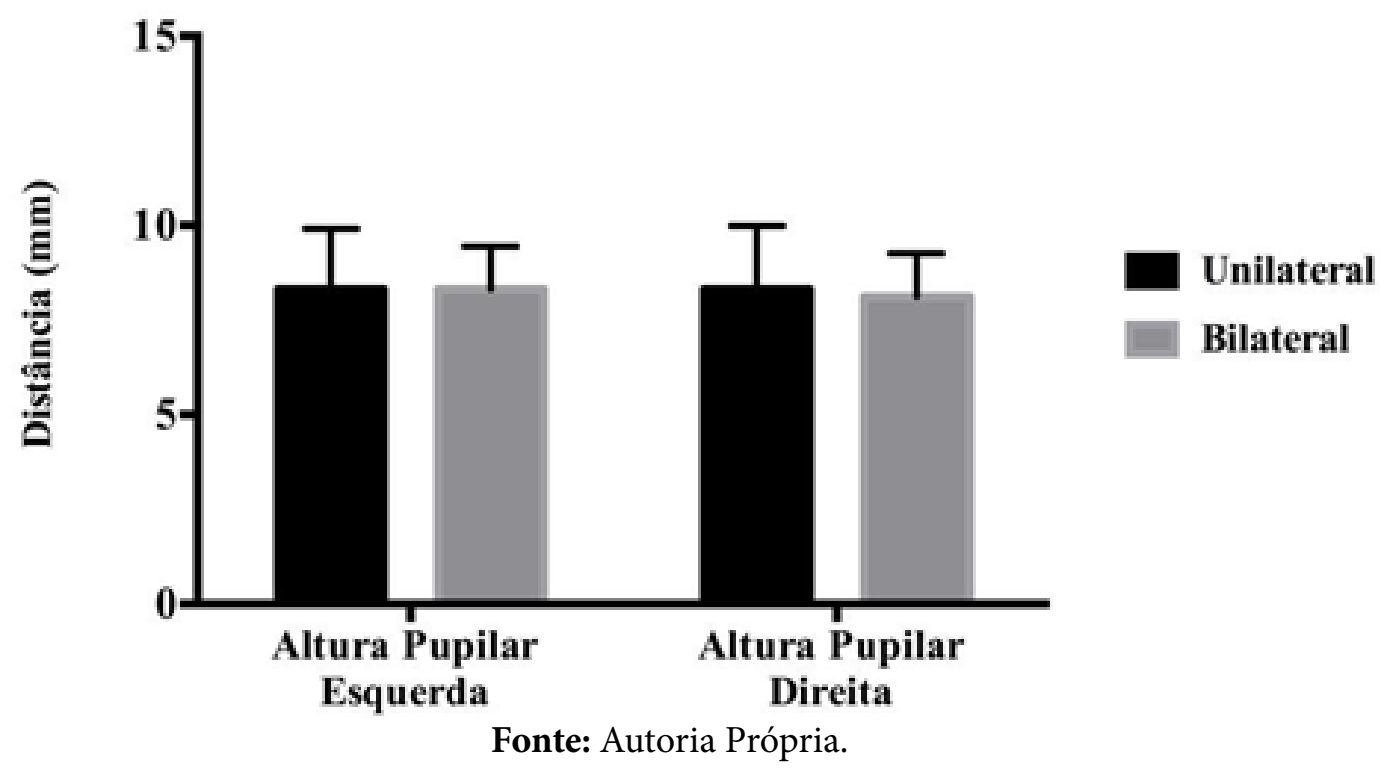

Figura 6 - Análise da mensuração linear do ângulo.

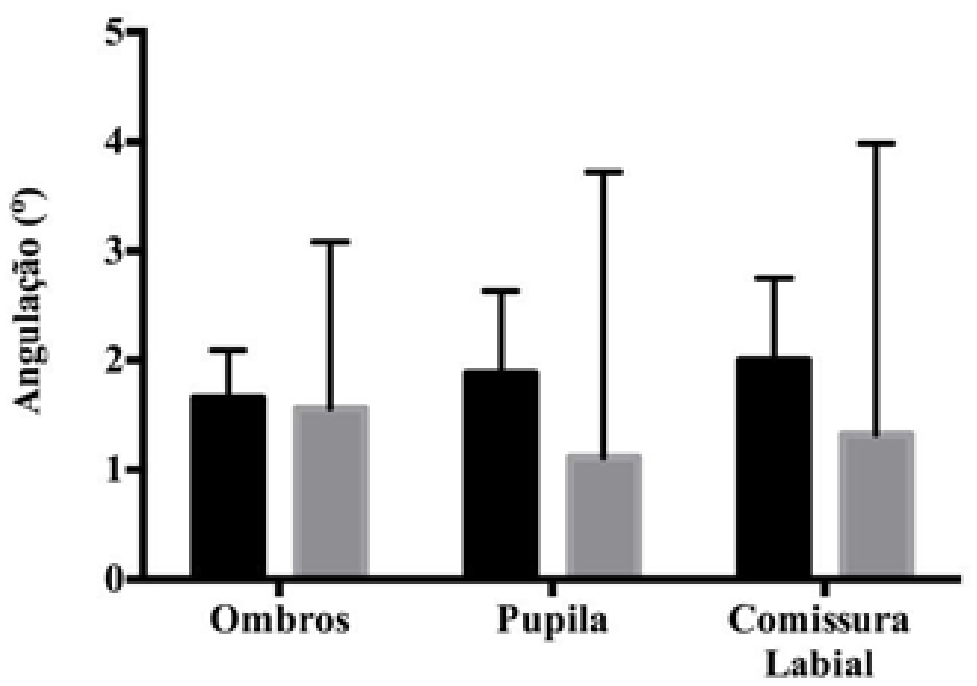

Fonte: Autoria Própria.

O teste do qui-quadrado revelou que houve diferença estatisticamente significante entre os grupos de mastigação bilateral e unilateral quanto à postura de ombro, cabeça e AFMP (respectivamente $\mathrm{p} \leq 0,05$ ). Por meio do coeficiente de contingência, foi encontrada forte correlação entre o padrão mastigatório e a postura de ombro $(\mathrm{CC}=0,510)$, de cabeça $(\mathrm{CC}=0,673)$ e também com o AFMP $(\mathrm{CC}=0,747)$ (Tabelas $1,2$ e 3$)$. 
Tabela 1 - Frequência de indivíduos para as categorias de postura de ombro segundo o padrão mastigatório.

\begin{tabular}{cccccc}
\hline & & \multicolumn{3}{c}{ OMBRO $^{* 1}$} & Total \\
& & Bem posicionado & Direito mais alto & Esquerdo mais alto & $\mathrm{n}(\%)$ \\
& bilateral & $12(48,0)^{\mathrm{a}}$ & $1(4,0)^{\mathrm{b}}$ & $12(48,0)^{\mathrm{b}}$ & $25(100,0)$ \\
\hline \multirow{2}{*}{ Mastigação } & direita & $0(0,0)^{\mathrm{a}}$ & $1(14,3)^{\mathrm{a}}$ & $6(85,7)^{\mathrm{a}}$ & $7(100,0)$ \\
& esquerda & $0(0,0)^{\mathrm{a}}$ & $3(37,5)^{\mathrm{b}}$ & $5(62,5)^{\mathrm{a}, \mathrm{b}}$ & $8(100,0)$ \\
& $12(30,0)$ & $5(12,5)$ & $23(56,5)$ & $40(100,0)$ \\
\hline
\end{tabular}

Fonte: Autoria Própria.

${ }^{*}$ significância pelo teste do qui-quadrado $=0,007$

${ }^{1}$ significância do coeficiente de contingência $=0,510$

a,b letras iguais significam que as proporções da coluna não diferem entre si considerando significância de 0,05 (z residual).

Tabela 2 - Frequência de indivíduos para as categorias de postura de cabeça segundo o padrão mastigatório.

\begin{tabular}{|c|c|c|c|c|c|}
\hline & & \multicolumn{3}{|c|}{ CABEÇA $^{* 1}$} & \multirow{2}{*}{$\begin{array}{l}\text { Total } \\
\text { n (\%) }\end{array}$} \\
\hline & & $\begin{array}{c}\text { Bem posicionada } \\
\mathrm{n}(\%)\end{array}$ & $\begin{array}{c}\text { Inclinada para direita } \\
\text { n (\%) }\end{array}$ & $\begin{array}{c}\text { Inclinada para esquerda } \\
\text { n (\%) }\end{array}$ & \\
\hline \multirow{3}{*}{ Mastigação } & bilateral & $20(80,0)^{\mathrm{a}}$ & $5(20,0)^{b}$ & $0(0,0)^{\mathrm{b}}$ & $25(100,0)$ \\
\hline & direita & $0(0,0)^{\mathrm{a}}$ & $7(100,0)^{\mathrm{b}}$ & $0(0,0)^{a, b}$ & $7(100,0)$ \\
\hline & esquerda & $0(0,0)^{\mathrm{a}}$ & $5(62,5)^{b}$ & $3(37,5)^{c}$ & $8(100,0)$ \\
\hline \multicolumn{2}{|c|}{ Total n (\%) } & $20(50,0)$ & $17(42,5)$ & $3(7,5)$ & $40(100,0)$ \\
\hline
\end{tabular}

Fonte: Autoria Própria.

* significância pelo teste do qui-quadrado $=0,000$

${ }^{1}$ significância do coeficiente de contingência $=0,673$

a,b,c letras iguais significam que as proporções da coluna não diferem entre si considerando significância de 0,05 ( $\mathrm{z}$ residual). 
Tabela 3 - Frequência de indivíduos para as categorias de ângulo de planas segundo o padrão mastigatório.

\begin{tabular}{|c|c|c|c|c|c|}
\hline & & \multicolumn{3}{|c|}{ ÂNGULO DE PLANAS ${ }^{* 1}$} & \multirow{2}{*}{$\begin{array}{l}\text { Total } \\
\text { n (\%) }\end{array}$} \\
\hline & & $\begin{array}{c}\text { Semelhante angulação } \\
\mathrm{n}(\%)\end{array}$ & $\begin{array}{c}\text { Menor ângulo direito } \\
\mathrm{n}(\%)\end{array}$ & $\begin{array}{c}\text { Menor ângulo esquerdo } \\
\mathrm{n}(\%)\end{array}$ & \\
\hline \multirow{3}{*}{ Mastigação } & bilateral & $18(72,0)^{\mathrm{a}}$ & $6(24,0)^{b}$ & $1(4,0)^{b}$ & $24(100,0)$ \\
\hline & direita & $0(0,0)^{\mathrm{a}}$ & $7(100,0)^{b}$ & $0(0,0)^{\mathrm{a}}$ & $7(100,0)$ \\
\hline & esquerda & $0(0,0)^{\mathrm{a}}$ & $0(0,0)^{\mathrm{a}}$ & $8(100,0)^{b}$ & $8(100,0)$ \\
\hline \multicolumn{2}{|c|}{ Total n (\%) } & $18(75,0)$ & $13(32,5)$ & $9(22,5)$ & $40(100,0)$ \\
\hline
\end{tabular}

Fonte: Autoria Própria.

* significância pelo teste do qui-quadrado $=0,000$

${ }^{1}$ significância do coeficiente de contingência $=0,747$

a,b letras iguais significam que as proporções da coluna não diferem entre si considerando significância de 0,05

( $\mathrm{z}$ residual).

Além disso, para todos os critérios da análise de RDC/TMD realizada, os critérios de diagnóstico de desarranjos de disco (Grupo 2) e desvio da linha média, apresentaram diferença nas frequências relatadas entre os grupos, sendo o considerado "normal" mais frequente nos pacientes com mastigação bilateral (Tabela 4). 
Tabela 4 - Análise da frequência de Critérios de Diagnóstico para Pesquisa das Disfunções Temporomandibulares (RDC/TMD).

\begin{tabular}{|c|c|c|c|}
\hline \multirow[t]{2}{*}{ Critérios de Diagnóstico } & \multicolumn{2}{|c|}{ Mastigação } & \multirow{2}{*}{$\begin{array}{c}\text { Valor de } \\
\text { "p" }\end{array}$} \\
\hline & Unilateral $(n=15)$ & Bilateral $(n=25)$ & \\
\hline Idade (Média; Mínimo - Máximo) & $24.8(19-41)$ & $24.2(17-40)$ & \\
\hline Limitações na função mandibular (\%) & $0.09(0.0-58.0)$ & $03.70(0.0-50.0)$ & \\
\hline \multicolumn{4}{|l|}{ Grupo 1 - n (\%) } \\
\hline Nenhum & $13(86.67)$ & $24(96.00)$ & $0.2779(N S)$ \\
\hline Dor Miofascial & $02(13.33)$ & $01(04.00)$ & \\
\hline \multicolumn{4}{|l|}{ Grupo 2 - n (\%) } \\
\hline Nenhum & $\begin{array}{c}10(66.67) \\
02(13.33)\end{array}$ & $\begin{array}{l}22(88.00) \\
\quad 02(08.00)\end{array}$ & $0.0378(S)$ \\
\hline Deslocamento do Disco com redução $\mathrm{D}$ & $02(13.33)$ & $02(08.00)$ & \\
\hline Deslocamento do Disco com redução E & $00(00.00)$ & $01(04.00)$ & \\
\hline Deslocamento do Disco com redução D e E & $02(13.33)$ & $00(00.00)$ & \\
\hline Deslocamento do Disco sem redução & $01(06.67)$ & $00(00.00)$ & \\
\hline \multicolumn{4}{|l|}{ Grupo 3 - n (\%) } \\
\hline Nenhum & $11(73.33)$ & $22(88.00)$ & $0.6534(N S)$ \\
\hline Artralgia esquerda & $03(20.00)$ & $01(04.00)$ & \\
\hline Artralgia direita & $00(00.00)$ & $00(00.00)$ & \\
\hline Artralgia esquerda e direita & $01(06.67)$ & $02(08.00)$ & \\
\hline \multicolumn{4}{|l|}{ Graduação da Dor - n (\%) } \\
\hline 0 & $09(60.00)$ & $19(76.00)$ & 0.1815 (NS) \\
\hline $\mathbf{I}$ & $00(00.00)$ & $00(00.00)$ & \\
\hline II & $03(20.00)$ & $05(20.00)$ & \\
\hline III & $03(20.00)$ & $01(04.00)$ & \\
\hline \multicolumn{4}{|l|}{ Depressão - n (\%) } \\
\hline Normal & $06(40.00)$ & $15(60.00)$ & 0.1625 (NS) \\
\hline Moderado & $05(33.33)$ & $07(28.00)$ & \\
\hline Grave & $04(26.67)$ & $03(12.00)$ & \\
\hline \multicolumn{4}{|l|}{ Sintomas físicos - n (\%) } \\
\hline Normal & $09(60.00)$ & $12(48.00)$ & 0.6094 (NS) \\
\hline Moderado & $00(00.00)$ & $01(04.00)$ & \\
\hline Grave & $06(40.00)$ & $12(48.00)$ & \\
\hline \multicolumn{4}{|l|}{ Desvio de linha média - n (\%) } \\
\hline Normal & $06(40.00)$ & $22(88.00)$ & $0.0111(S)$ \\
\hline Inferior desviada para esquerda & $07(46.67)$ & $02(08.00)$ & \\
\hline Inferior desviada para direita & $01(06.67)$ & $01(04.00)$ & \\
\hline Superior desviada para direita & $01(06.67)$ & $00(00.00)$ & \\
\hline \multicolumn{4}{|l|}{ Guias - n (\%) } \\
\hline Guia do Canino & $13(86.67)$ & $20(80.00)$ & $0.5911(\mathrm{NS})$ \\
\hline Função em grupo & $02(13.33)$ & $05(20.00)$ & \\
\hline
\end{tabular}

Fonte: Autoria Própria. 


\section{Discussão}

O método de identificação do padrão mastigatório utilizado foi a visualização direta com 1 ou mais examinadores treinados (MIOCHE et al., 1999; PIGNATARO, BERZIN, RONTANI, 2013; LAMONTAGNE, AL-TARAKEMAH, HONKALA, 2013) com associação da filmagem (LUCENA et al., 2006; PEREIRA JUNIOR, FAVILLA, DWOKIN, 2004). Apesar do método mais comumente encontrado na literatura ser a eletromiografia de superfície dos músculos mastigatórios (MIOCHE et al., 1999; SHIGA et al., 2003; PIGNATARO, BERZIN; RONTANI, 2013) a opção pelo método visual associado à comprovação da filmagem foi embasada no estudo de PIGNATARO; BÉRZIN, RONTANI (2004) que comprovou eficácia e confiabilidade similar de ambos os métodos. Na tentativa de minimizar as falhas na identificação do padrão mastigatório foram utilizados 3 tipos de alimentos, bala de goma, amêndoa e goma de mascar, com propriedades viscoelásticas e texturas diferentes. A literatura sugere diferentes padrões de mastigação com o uso de diferentes alimentos (CAZAL, 2016). A textura do alimento modifica a força mastigatória e os movimentos mandibulares, o comportamento dos músculos mastigatórios, a duração dos ciclos mastigatórios e o número de ciclos que precedem a primeira deglutição (MIOCHE et al., 1999). A amêndoa, por ser um alimento mais resistente e que necessita de maior força mastigatória no início do ciclo (MIOCHE et al., 1999; VAN DER BILT; ABBINK, 2017), foi utilizada para evidenciar o lado preferencial mastigatório. Já a goma de mascar (Trident) foi utilizada por estimular movimentos mastigatórios mais estáveis, como previamente descrito por SHIGA et al. (2003).

A falta de consenso na literatura (LAGAIDA; WHITE, 1983; POIKELA, PIRTTINIEMI; KANTOMA, 2000; CASTRO et al., 2003) quanto a relação entre a assimetria facial e a mastigação unilateral, estimulou a inclusão da análise facial na metodologia deste estudo. Nesta análise, a assimetria facial foi verificada e quantificada diante da presença de uma diferença existente entre a distância da pupila ao plano labial do lado direito e do esquerdo. Ao comparar o grupo que apresentava mastigação unilateral com o grupo de mastigação bilateral, não foi observada diferença estatística significante entre os grupos. Tal resultado corrobora com o estudo de CASTRO et al. (2003) que também não encontrou nenhuma diferença estatística quanto a análise facial de assimetria. Contudo, os resultados confrontam com os achados de LAGAIDA e WHITE (1983) na qual investigaram alterações da simetria facial em pacientes com mastigação unilateral, em uma amostra de vinte jovens de 7 a 11 anos, e concluíram que a mastigação unilateral afeta as características faciais dos pacientes. Observaram ainda que a altura pupilar é maior do lado de mastigação unilateral em razão da dimensão vertical nesse lado; enquanto que o lado de balanceio mostrou-se menos desenvolvido. Estes achados também foram observados em um estudo in vivo desenvolvido por POIKELA, PIRTTINIEMI e KANTOMA, 2000, que ao induzir a mastigação unilateral em coelhos em fase de crescimento verificou uma alteração no crescimento mandibular, no formato da fossa glenóide, além de alterações histoquímica que comprometeram a simetria facial. As divergências observadas entre os resultados citados podem ter ocorrido devido às diferentes faixas etárias dos estudos, uma vez que em alguns foram utilizadas amostras de indivíduos jovens (em fase de crescimento) e em outras, indivíduos adultos. Se a mastigação unilateral ocorre ainda durante a fase de crescimento, podemos considerar que a mesma aumenta a possibilidade de ocorrer uma assimetria facial mais evidente.

Apesar do estudo de SHIMAZAKI et al. (2003), utilizando a análise de elementos finitos, comprovar que tanto a inclinação do plano oclusal quanto a falta de simetria na contração dos músculos mastigatórios (semelhante ao que ocorre na mastigação unilateral) atuam no deslocamento da coluna cervical como um controle compensatório da postura, esta associação não foi encontrada em nosso estudo.

Tanto o AFMP como a presença da disfunção temporomandibular (DTM) foram comprovadamente relacionadas com a mastigação unilateral. Resultado semelhante ao de PIGNATARO NETO (2000) que obteve uma associação de 75\% do AFMP com a mastigação unilateral.

Ao verificar a associação entre DTM e o lado de preferência mastigatória, ficou evidente que pacientes 
apresentando mastigação bilateral apresentaram menor sintomatologia relacionada à disfunção, fato este, já bem esclarecido na literatura, a qual demonstra uma evidente associação entre a mastigação unilateral e sinais e sintomas da DTM (FERRARIO et al., 2001;REINHARDT et al., 2006; SANTANA-MARA et al., 2013; WEBER et al., 2013). Segundo WEBER et al. (2013), indivíduos com DTM apresentam maior esforço dos lábios e interposição lingual durante a deglutição, e maior distância entre o osso hióide e a mandíbula.

\section{Conclusão}

Pode-se concluir que a mastigação unilateral apresenta uma associação com a presença de sinais e sintomas da DTM, com desvios na linha média e com alterações na postura de ombro, cabeça e AFMP.

\section{REFERÊNCIAS}

BEZERRA, L.A. Lado de preferência mastigatória e suas relações com a atividade elétrica muscular, postura e descarga de peso podal em crianças. 2018. Tese de Doutorado - Neuropsiquiatria e Ciência do Comportamento, Universidade Federal de Pernambuco, Recife, 2018.

BHATKA, R.; THROCKMORTON, G.S.; WINTERGERST, A.M.; HUTCHINS, B.; BUSCHANG, P.H. Bolus size and unilateral chewing cycle kinematics. Arch Oral Biol, v.49, n.7, p. 559-66, 2004.

CAMPOS, T.N.; FUKUMOTO, L.Y.; IFUKO, F.Y.; HARA, E.S.; TIBA, H.S.; MORI, M. Influência da desoclusão posterior unilateral no equilíbrio corporal de ratos. Rev. Odont. da Universidade Cidade de São Paulo, v.20, n.1, p.30-6, jan-abr 2008.

CASANOVA-ROSADO, J.F.; MEDINA-SOLÍS, C.E.; VALLEJOS-SÁNCHEZ, A.A.; CASANOVA-ROSADO, A.J.; HERNÁNDEZ-PRADO, B.; AVILA-BURGOS, L. Prevalence and associated factors for temporomandibular disorders in a group of Mexican adolescents and youth adults. Clin Oral Investig, v.10, n.1, p.42-49, 2006.

CASTRO AV. Estudo das alterações morfológicas mandibulares e faciais ocasionadas pela mastigação unilateral 2003. Dissertação (Mestre em Odontologia-área de concentração Ortodontia)- Faculdade de Odontologia da PUC-MG, Belo Horizonte, 2003.

CAZAL, M.S.; DA SILVA, A.M.; GALO, R.; MESTRINER JUNIOR, W.; DA SILVA, M.A. Comparison of dynamic electromyographic analysis of masticatory capsules with materials of different textures. Cranio, v.34, n.2, p.105-111, 2016.

CUCCIA, A.; CARADONNA, C. The relationship between the stomatognathic system and body posture. Clinics, v.64, n.1, p.61-6, 2009.

D’ATTILIO, M.; FILIPPI, M.R.; FEMMINELLA, B.; FESTA, F.; TECCO, S. The influence of an experimentally-induced malocclusion on vertebral alignment in rats: a controlled pilot study Cranio, v.23, n.2, p.119-29, Apr. 2005.

DWORKIN, S.F.; LE RESCHE, L. Research diagnostic criteria for temporomandibular disorders: review, criteria, examinations and specifications, critique. J Craniomandib Disord, v.6, n.4, p.301-55, 1992. 
FASSICOLLO, C.E. Análise da coordenação e sincronia dos músculos da mastigação e do pescoço durante a mastigação de pacientes com Desordem Temporomandibular crônica Tese (Faculdade de Medicina de Ribeirão Preto) -Universidade de São Paulo, Ribeirão Preto, 2020.

FEDIV, I. Avaliação do ângulo funcional mastigatório e as suas implicações clínicas. Dissertação (Faculdade de Medicina dentária)- Universidade Católica Portuguesa,Viseu, 2017.

FELÍCIO, C.M.; COUTO, G.A.; FERREIRA, C.L.P.; MESTRINER-JUNIOR, W. Confiabilidade da eficiência mastigatória com beads e correlação com atividade muscular. Pró- Fono, v.20, n.4, p.225-30, 2008.

FELÍCIO, C.M.; MELCHIOR, M.O.; SILVA, M.A. Effects of orofacial myofunctional therapy on temporamanbilar disorders. Cranio, v.28, n.4, p.249-59, 2010.

FERRARIO, V.F.; SFORZA, C.; SCHMITZ, J.H.; SERRAO, G. Comparison of unilateral chewing movements vs. dental guidance through the dental guidance ratio. J Prosthet Dent, v.86, n.6, p.586-91, 2001.

FERREIRA, C.L.P.; SILVA, M.A.M.R.; FELÍCIO, C.M. Orofacial Myofunctional Disorder in Subjects with temporomandibular Disorder. The journal of craniomandibular practice, v.27, n.4, p.268-74, 2009.

FUJITA, Y.; MOTEGI, E.; NOMURA, M.; KAWAMURA, S.; YAMAGUCHI, D.; YAMAGUCHI, H. Oral habits of temporomandibular disordens patients with malocclusion. Bull. Tokyodent, v.44, n.4, p.201-7, 2003.

HIGILEH, Y.A. Interligações Posturais e Mandíbulares. Dissertação (Departamento de ciências médicas)- Universidade Fernando Pessoa, Porto, 2019.

KHAN, K.S.; KUNZ, R.; KLEIJNEN, J.; GERD A. Five steps to conducting a systematic review. J R Soc Med, v.96, n.3, p.118-121, 2003.

KIBANA, Y.; ISHIJIMA, T.; HIRAI, T. Occlusal support and head posture. J Oral Rehabil, v.29, n.1, p.58-63, Jan.2002.

KOHNO, S.; YOSHIDA, K.; KOBAYASHI, H. Pain in the sternocleidomastoid muscle and occlusal interferences. J. Oral Rehabil, v.15, n.4, p.385-92, Jul.1988.

KUMAI, T. Difference in chewing patterns between involved and opposite sides in patients with unilateral temporomandibular joint and mysfacial paint-dysfunction. Archs oral Biol, v.38, n.6, p.467-78, 1993.

KURNIA, S.I.; HIMAWAN, L.S.; TANTI, I.; ODANG, R.W. Correlation between Chewing Preference and Condylar Asymmetry in Patients with Temporomandibular Disorders. J. Phys.: Conf. Ser, v.1073, n.3, 2018.

LAGAIDA, M.E.; WHITE, G.E. Unilateral mastication and facial formation. J Pedod, v.7, n.2, p.127-34, 1983.

LAMONTAGNE, P.; AL-TARAKEMAH, Y.; HONKALA, E. Relationship between the preferred chewing 
Castilho et al.

side and the angulation of anterior tooth guidance. Med Princ Pract, v.22, n.1, p.545-9, 2013.

LA TOUCHE, R.; LOSANA-FERRER, A.; PASCUAL-VAQUERIZO, E.; SUSO-MARTÍ, L.; PARIS-ALEMANY, A.; CHAMORRO-SÁNCHEZ, J. et al. Orofacial sensorimotor behaviour in unilateral chewing: A comparative analysis in asymptomatic population. Physiology \& Behavior 2 v.212, n.1, p.112718, December 2019.

LIST, T.; DWORKIN, S.F. Comparing TMD diagnoses and clinical findings at Swedish and US TMD centers using research diagnostic criteria for temporomandibular disorders. J Orofac Pain, v.10, n.3, p.240-53, 1996.

LUCENA, L.B.; KOSMINSKY, M.; COSTA, J.; GÓES, P.S. Validation of the Portuguese version of the RDC/TMD Axis II questionnaire. Braz Oral Res, v.20, n.4, p.312-17, 2006.

MARTINEZ-GOMIS, J.; LUJAN-CLIMENT, M.; PALAU, S.; BIZAR, J.; SALSENCH, J.; PERAIRE, M. Relationship between chewing side preference and handedness and lateral asymmetry of peripheral factors. Archives of oral biology, v.54, n.1, p.101-107, 2009.

MICHELOTTI, A.; BUONCORE, G.; MANZO, P.; PELLEGRINO, G.; FARELLA, M. Dental occlusion and posture: an overview. Prog Orthod, v.12, n.1, p.53-8, 2011.

MIOCHE, L.; BOURDIOL, P.; MARTIN, J.F.; NOËL, Y. Variations in human masseter and temporalis muscle activity related to food texture during free and side-imposed mastication. Arch Oral Biol, v.44, n.12, p.1005-12, 1999.

MIYAKE, R.; OHKUBO, R.; TAKEHARA, J.; MORITA, M. Oral prafunctions and association with symptoms of temporomandibular disorders in Japanese university students. J Oral Rehabil, v.6, n.31, p.518-23, Jun 2004.

MIYAWAKI, S.; OHKOCHI, N.; KAWAKAMI, T.; SUGIMURA, M. Changes in masticatory muscle activity according to food size in experimental human mastication. J Oral Rehabil, v.28, n.8, p.778-84, 2001.

PASINATO, F.; SANTOS-COUTO-PAZ, C.C.; ZEREDO, J.L.L.; MACEDO, S.B.; CORREAA, E.C.R. Experimentally induced masseter-pain changes masseter but not sternocleidomastoid muscle-related activity during mastication. Journal of Electromyography and Kinesiology, v.31, p.88-95, Dec.2016.

PEREIRA JÚNIOR, F.J.; FAVILLA, E.E.; DWORKIN, S.F. Critérios de diagnóstico para pesquisa das disfunções temporomandibulares (RDC/TMD). Tradução oficial para a língua portuguesa. Bras Clin Odontol Integr, v.8, n.47, p.384-95, 2004.

PIGNATARO NETO, G.; BÉRZIN, F.; RONTANI, R.M.P. Identificação do lado de preferência mastigatória através de exame eletromiográfico comparado ao visual. R Dental Press Ortop Facial, v.9, n.4, p.77-85, 2004.

PIGNATARO NETO, G. Análise da correlação dos ângulos funcionais mastigatórios direito e es- 
querdo com o lado de preferência mastigatória. Dissertação (Mestrado) - Faculdade de Odontologia - Unicamp, Piracicaba, 2000.

POIKELA, A.; KANTOMAA, T.; PIRTTINIEMI, P. Cranio facial growth after a period of unilateral masticatory function in young rabbits. Eur J Oral, v.105, n.1, p.331-7, 1997.

POIKELA, A.; PIRTTINIEMI, P.; KANTOMAA, T. Location of the glenoid fossa after a period of unilateral masticatory function in young rabbits. Eur J Oral, v.22, n.1, p.105-12, 2000.

REINHARDT, R.; TREMEL, T.; WEHRBEIN, H.; REINHARDT, W. The Unilateral Chewing Phenomenon, Occlusion and TMD. The journal of crânio mandibular practice, v,24, n.1, p.166-70, 2006.

RILO, B.; FERNÁNDEZ-FORMOSO, N.; MORA., M.J.; CADARSO-SUÁREZ, C.; SANTANA, U. Distance of the contact glide in the closing masticatory stroke during mastication of three types of food. $\mathbf{J}$ Oral Rehabil, v.36, n.8, p.571-6, 2009.

ROVIRA-LASTRA, B.; FLORES-OROZCO, E.I.; SALSENCH, J.; PERAIRE, M.; MARTINEZ-GOMIS, J. Is the side with the best masticatory performance selected for chewing. Arch Oral Biol,.v.59, n.12, p.1316-20, 2014.

ROVIRA-LASTRA, B.; FLORES-OROZCO, E.I.; AYUSO-MONTERO, R.; PERAIRE, M.; MARTINEZ-GOMIS, J. Peripheral, functional and postural asymmetries related to the preferred chewing side in adults with natural dentition. J Oral Rehabil, v.43, n.4, p.279-85, Apr 2016.

SANTANA-MORA, U.; LÓPEZ-CEDRÚN, J.; MORA, M.J.; OTERO, X.L.; SANTANA-PENÍN, U. Temporomandibular disorders: the habitual chewing side syndrome. Plos One, v.8, n.4, e59980, 2013.

SHIGA, H.; KOBAYASHI, Y.; ARAKAWA, I.; SHONAI, Y. Selection of food and chewing side for evaluating masticatory path stability. Odontology, v.91, p.26-30, 2003.

SHIMAZAKI, T.; MOTOYOSHI, M; HOSOI, K; NAMURA, S. The effect of occlusal alteration and masticatory imbalanceon the cervical spine. European Journal of Orthodontics, v.25, n.1, p.457-63, 2003.

SOBOL,EVA, U.; LAURIN̦A, L.; SLAIDIN̦A, A. The masticatory system - an overview. Stomatologija, Baltic Dental and Maxillofacial Journal, v.7, p.77-80, 2005.

TIWARI, S.; NAMBIAR, S.; UNNIKRISHNAN, B. Chewing side preference- Impact on facial symmetry, dentition and temporomandibular joint its correlation with handedness. J Orofac Sci, v.9, p.22-7, 2017.

UEDA, T.; SAKURAI, K.; SUGIYAMA, T. Individual difference in the number of chewing strokes and its determinant factors. J Oral Rehabil, v.33, n.2, p.85-93, 2006.

VAN DER BILT, A.; ABBINK, J.H. The influence of food consistency on chewing rate and muscular work. Arch Oral Biol, v.83, p.105-110, 2017.

VAN DER BILT, A.; ENGELEN, L.; PEREIRA, L.J.; VAN DER GLAS, H.E.; ABBINK, J.H. Oral physiolo- 


\section{Castilho et al.}

gy and mastication. Physiology \& Behavior, v.89, p.22-27, 2006.

WEBER, P.; CORRÊA, E.C.R.; BOLZAN, G.P.; FERREIRA, F.S.; SOARES, J.C.; SILVA, A.M.T. Mastigação e deglutição em mulheres jovens com desordem temporomandibular. Codas, v.25, n.4, p.375-80, 2013.

YAMAGUCHI, S.; ITOH, S.; WATANABE, Y.; TSUBOI, A.; WATANABE, M. Quantitative analysis of masticatory activity during unilateral mastication using muscle fMRI. Oral Dis, v.17, n.4, p.40713, 2011. 